\title{
ATIVIDADE ANTIOXIDANTE DE ÓLEOS ESSENCIAIS DE ESPÉCIES DE Croton DO NORDESTE DO BRASIL
}

\author{
Selene Maia de Morais*, Francisco Eduardo Aragão Catunda Júnior, Ana Raquel Araújo da Silva e Jason Stone \\ Martins Neto \\ Curso de Química, Universidade Estadual do Ceará, Av. Paranjana, 1700, Campus do Itaperi, 60740-000 Fortaleza - CE \\ Davide Rondina \\ Faculdade de Veterinária, Universidade Estadual do Ceará, Av. Paranjana, 1700, Campus do Itaperi, 60740-000 Fortaleza - CE \\ José Henrique Leal Cardoso
}

Instituto de Ciências Biomédicas, Universidade Estadual do Ceará, Av. Paranjana, 1700, Campus do Itaperi, 60740-000

Fortaleza - CE

Recebido em 10/3/05; aceito em 4/11/05; publicado na web em 5/5/06

\begin{abstract}
ANTIOXIDANT ACTIVITY OF ESSENTIAL OILS FROM NORTHEASTERN BRAZILIAN CROTON SPECIES. Three Croton species, C. zenhtneri, C. nepetaefolius and C. argyrophylloides, were collected at two different times, 6:00 and 13:00 h, their essential oils were extracted by steam distillation and analyzed by gas Chromatography / Mass Spectrometry. The percentage yield of oil constituents changes along the day. The oils were submitted to the antioxidant test thiobarbituric acid reactive species, using BHT and a-tocoferol as the reference compounds. All oils exhibited good antioxidant activities. In general, C. zenhtneri and $C$. argyrophylloides essential oils showed higher antioxidant activity than C. nepetaefolius.
\end{abstract}

Keywords: essential oil; antioxidant; TBARS test.

\section{INTRODUÇÃ̃O}

O ecossistema Caatinga cobre quase 1 milhão de $\mathrm{km}^{2}$ no Nordeste do Brasil. A vegetação xerófila característica é afetada pela longa e irregular seca, altas temperaturas e elevada radiação ultravioleta. Principalmente devido às famílias de baixa renda, o uso de plantas medicinais e auto-medicação é grandemente estendido na região ${ }^{1}$.

Nas plantas, a síntese de metabólitos secundários antioxidantes que absorvem em 300-400 nm é significantemente aumentada por radiação $\mathrm{UV}^{2}$ fornecendo, portanto, um alto nível de proteção contra oxidantes prejudiciais gerados termicamente ou pela luz ${ }^{3}$. Além disso, embora plantas medicinais sejam raramente utilizadas como antioxidantes em medicina tradicional, suas características terapêuticas poderiam ser sustentadas devido, em parte, a sua capacidade varredora de radicais livres que podem estar envolvidos em muitas doenças ${ }^{4}$. Câncer, enfisema, cirrose, arterosclerose e artrites têm sido correlacionados com stress oxidativo. Os organismos em geral são protegidos contra os danos causados pelos radicais livres, por enzimas como superóxido desmutase e catalase, ou compostos como ácido ascórbico, tocoferol e glutationa. Quando os mecanismos da proteção antioxidante se tornam ineficientes por fatores como idade, a deterioração das funções fisiológicas pode ocorrer, resultando em doenças e aceleração do envelhecimento. Entretanto, suplementos alimentares antioxidantes podem ser usados para ajudar o corpo humano a reduzir os danos oxidativos 5 .

Os óleos, as gorduras e os alimentos que os contêm estão sujeitos, durante o processamento e a estocagem, a reações químicas que podem alterar, de modo indesejável, as características do produto final. Hidrólises e oxidações podem ser responsáveis por esse processo, sobretudo a oxidação dos lipídeos. Antioxidantes são geralmente utilizados em óleos e comidas gordurosas para retardar sua autooxidação ${ }^{6}$. Alguns antioxidantes sintéticos como butil-hidroxianisol (BHA) e butil-

*e-mail: selene@uece.br hidroxitolueno (BHT), bastante utilizados em alimentos, revelaramse tóxicos em altas doses ${ }^{7}$. Desta forma, a pesquisa por antioxidantes naturais tem aumentado bastante nos últimos anos ${ }^{8,9}$.

Óleos essenciais são constituintes voláteis orgânicos responsáveis pela fragrância de muitas plantas. Podem ser obtidos de flores, folhas, frutos, sementes, gramas, raízes, rizomas e caules das plantas $^{10}$. A atividade antioxidante dos óleos voláteis tem sido bastante estudada ${ }^{11,12}$ e os óleos essenciais de plantas do Nordeste brasileiro constituem fontes potenciais de antioxidantes naturais ${ }^{13}$.

Croton zehntneri Pax et Hoffm., conhecida popularmente como "canela de cunhã", "canelinha" ou "canela-brava", é uma planta subarbustiva e caducifólia do Nordeste brasileiro, cujas folhas e talos são dotadas de um aroma que lembra uma mistura de erva-doce e cravo-da-Índia. Entretanto, este aroma mostra-se variável entre exemplares desta planta coletados em diferentes localidades do Nordeste. Isto se deve à variação na concentração dos constituintes químicos mais abundantes nos seus óleos essenciais ${ }^{14}$. Assim, distinguem-se para esta espécie quatro tipos químicos como: anetol - para os exemplares coletados em Fortaleza (CE) e Viçosa (CE); eugenol para os coletados em Areia Branca (RN) e Quixadá (CE); metileugenol - para os coletados em Ipu (CE) e Oeiras (PI); estragol para os exemplares coletados em Tianguá (CE) e Granja (CE).

O Croton zehntneri é usado na medicina popular principalmente como sedativo, como estimulante de apetite e para aliviar distúrbi-
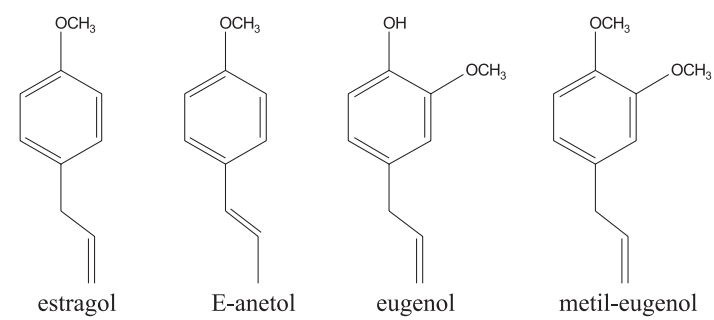

Esquema 1. 
os intestinais, sendo comprovados os efeitos antinociceptivos de seu óleo essencial ${ }^{15}$.

O Croton nepetaefolius é largamente utilizado na medicina popular como estomáquico, carminativo e para cólicas intestinais, sendo esta última ação comprovada cientificamente ${ }^{16}$. Não há informações do uso popular do Croton argyrophylloides conhecido como marmeleiro prateado devido ao aspecto de suas folhas.

Neste trabalho foi realizada a avaliação da atividade antioxidante dos óleos essenciais das três espécies do gênero Croton mencionadas, comuns no semi-árido nordestino: C. zehntneri, C. nepetaefolius e C. argyrophylloides, coletadas em dois horários diferentes do dia, utilizando o método TBAR's (quantificação das espécies reativas ao ácido tiobarbitúrico).

\section{PARTE EXPERIMENTAL}

\section{Coleta do material vegetal}

C. zenhtneri, C. nepetaefolius e C. argyrophylloides foram coletados (parte aérea) na Fazenda Cocalzinho, situada na cidade de Viçosa (CE), no dia 20/09/2002 e seus números de exsicatas são, respectivamente: $32.446,32.448$ e 32.444 , estando depositadas no Herbário Prisco Bezerra, da Universidade Federal do Ceará. Foram feitas coletas no mês de setembro em dois horários diferentes do dia - 6:00 e 13:00 h.

Reagentes utilizados: $\alpha$-tocoferol, 3,5-di-terc-butil-4-hidroxitolueno (butil hidroxytoluene-BHT), dodecil sulfato de sódio (SDS), DPPH (1,1-difenil-2-picril-hidrazila), ácido tiobarbitúrico (TBA) e o cloreto de 2,2'-azo-bis (2-amidinopropano) (ABAP), sendo todos de pureza analítica. Os quatro primeiros foram adquiridos junto à Sigma Chemical Co. (St. Louis, MO, EUA), o TBA, junto à Merck KgaA (Darmstadt, Alemanha) e o ABAP, junto à Wako Chemicals USA Inc. (Richmond, VA, EUA).

\section{Extração dos óleos essenciais}

Foi utilizado o método de destilação por arraste com vapor d'água em aparelho tipo Clevenger ${ }^{17}$. $\mathrm{O}$ tempo de extração foi de aproximadamente $2 \mathrm{~h}$ para cada planta. Os óleos essenciais foram em seguida secos com sulfato de sódio anidro e mantidos em refrigerador até a análise.

\section{Análise dos óleos essenciais - cromatografia com fase gasosa} acoplada à espectrometria de massa (CG/MS)

Foi realizada no Parque de Desenvolvimento Tecnológico do Ceará (PADETEC - UFC), através do método de cromatografia gasosa acoplada à espectrometria de massa (CG/EM) em um aparelho da Hewlett-Packard 5971. Condições programadas no aparelho: coluna capilar de DB-1 (dimetil-polisiloxane) com $30 \mathrm{~m}$ de comprimento e 0,25 mm de diâmetro interno; gás de arraste: Hélio $(1 \mathrm{~mL} / \mathrm{min})$; programa: $50-180{ }^{\circ} \mathrm{C}$ a $4{ }^{\circ} \mathrm{C} / \mathrm{min}$ e, depois, $180-280{ }^{\circ} \mathrm{C}$ a $20{ }^{\circ} \mathrm{C} / \mathrm{min}$; temperatura do injetor: $220{ }^{\circ} \mathrm{C}$; modo de injeção: $0,1 \mu \mathrm{L}$ (solução 10\%), split 1:20, $500 \mathrm{ng} / \mathrm{na}$ coluna. Os espectros de massas foram produzidos por impacto eletrônico $(70 \mathrm{eV})$. Os espectros de massas dos constituintes foram comparados com os padrões existentes na biblioteca Wiley do computador no aparelho. Em seguida, foram feitas comparações visuais com espectros de massa de substâncias encontrados na literatura ${ }^{18}$. Os tempos de retenção dos picos maiores, mais facilmente identificados pelo espectro de massa, foram comparados com os tempos de retenção destas substâncias registrados em catálogos ${ }^{18}$, observando-se a diferença que se manteve aproximadamente constante para as demais substâncias identifi- cadas pelo computador do aparelho. A coluna do cromatógrafo é similar (apolar) à relatada na referência utilizada.

\section{Teste de atividade antioxidante dos óleos essenciais - Método modificado do ácido tiobarbitúrico-espécies reativas ${ }^{19}$}

Usou-se, como meio rico em lipídeos, solução de gema de ovo homogeneizada. $\mathrm{O}$ procedimento foi feito em quintuplicata. Em cinco tubos de ensaio foram colocados $0,5 \mathrm{~mL}$ da solução de gema de ovo $(10 \% \mathrm{~m} / \mathrm{v})$ e $0,1 \mathrm{~mL}$ de solução da amostra (óleos essenciais e padrões) dissolvida em metanol, completando-se o volume para $1 \mathrm{~mL}$ com água. Foram testadas três concentrações para as soluções de cada amostra: 100, 500 e 1000 ppm (m/v). Em seguida, foi adicionado, a cada tubo, $0,05 \mathrm{~mL}$ de $\mathrm{ABAP}(0,07 \mathrm{~mol} / \mathrm{L})$ para induzir a peroxidação dos lipídeos. Adicionaram-se $1,5 \mathrm{~mL}$ de solução $20 \%$ de ácido acético (pH 3,5) e $1,5 \mathrm{~mL}$ de solução de TBA $(0,8 \%$ m/v) em solução de SDS $(1,1 \% \mathrm{~m} / \mathrm{v})$. Foi preparado, também, um tubo de ensaio com o experimento controle, no qual foram adicionados todos os reagentes, com exceção da amostra testada, para se observar a completa peroxidação dos lipídeos. Os tubos de ensaio foram levados ao banho-maria à 95 ${ }^{\circ} \mathrm{C}$, sob agitação, por $1 \mathrm{~h}$. Após resfriamento, foram adicionados $5 \mathrm{~mL}$ de 1-butanol a cada tubo e centrífugados a $3000 \mathrm{rpm}$, durante $10 \mathrm{~min}$. Mediu-se então a absorbância da camada orgânica superior em um espectrofotômetro Spekol no comprimento de onda de $532 \mathrm{~nm}$. Por fim, obteve-se o Índice Antioxidante da amostra em percentual (IA\%) usando a fórmula: IA\% $=(1-\mathrm{A} / \mathrm{C}) \times 100$, onde C é a absorbância do controle totalmente oxidado e A, a média aritmética das absorbâncias da amostra testada. Utilizou-se como padrão positivo neste método os antioxidantes BHT e $\alpha$-tocoferol nas mesmas proporções a que foram submetidos os óleos essenciais.

\section{Análise estatística}

Todos os cálculos foram feitos no programa de computador $\mathrm{SAS}^{20}$. Os dados foram submetidos à análise de variância (ANOVA), usando o procedimento GLM. As variações testadas foram as três concentraçoes em relação ao mesmo óleo e dentro da mesma concentração os diversos óleos e padrões. Para comparações entre as médias dos índices de atividade (IA\%), utilizou-se o teste de Duncan. As diferenças foram consideradas estatisticamente significantes qundo $\mathrm{P}<0,05$.

\section{RESULTADOS E DISCUSSÃO}

As análises dos óleos essenciais por cromatografia gasosa acoplada à espectrometria de massa estão mostradas nas Tabelas 1, 2 e 3 , registrando-se os principais constituintes químicos presentes nestes óleos. No óleo do C. zenhtneri coletado de manhã cedo (6:00 h), o $E$-anetol apresenta-se em maior percentual que o somatório do anisaldeído e o formiato de anisila, que são considerados biossinteticamente seus produtos de oxidação. Às 13:00 h estes dois produtos juntos representam um maior percentual. Pode-se supor que o maior teor de oxigênio e de radiação solar no ambiente às 13:00 h contribua para o aumento dos produtos oxidados. O óleo do C. nepetaefoluis apresenta o metileugenol como principal constituinte e sua concentração diminue às 13:00 h, enquanto o segundo componente mais abundante, o $\alpha$-copaeno, não mostra variação nos dois horários de coleta. No óleo do C. argyrophylloides, $\alpha$-pineno e 1,8-cineol diminuíram e espatulenol e cariofileno aumentaram.

Pela Tabela 4, verifica-se que os óleos essenciais obtidos nos dois horários de coletas das espécies do gênero Croton revelaram-se agentes antioxidantes relevantes. Todos os óleos essenciais apresentaram atividades antioxidantes que diminuíram 
Tabela 1. Composição química percentual do óleo essencial do Croton zehntneri Pax et Hoffm. coletado em dois horários diferentes do dia

\begin{tabular}{lcc}
\hline Constituintes & \multicolumn{2}{c}{ Horário de coleta } \\
\cline { 2 - 3 } & $6: 00 \mathrm{~h}$ & $13: 00 \mathrm{~h}$ \\
\hline$\alpha$-pineno & 3,62 & - \\
Canfeno & 1,58 & - \\
1,8 -Cineol & 1,83 & 2,12 \\
Cânfora & 2,15 & 2,69 \\
Estragol & - & 1,70 \\
para-Anisaldeído & 19,32 & 27,37 \\
$E$-Anetol & 47,34 & 30,97 \\
Formiato de anisila & 11,49 & 16,47 \\
$\beta$-Elemeno & 4,61 & 6,53 \\
$E$-Cariofileno & 1,22 & 2,65 \\
$\alpha-E$-Bergamoteno & 1,92 & 2,80 \\
$\alpha$-Guaieno & - & 1,93 \\
$\beta$-Selineno & 1,24 & 2,75 \\
$E$-Metil-isoeugenol & 1,38 & - \\
$\beta-$ Selineno & - & 2,42 \\
Óxido de cariofileno & 1,29 & 5,58 \\
\hline
\end{tabular}

Tabela 2. Composição química percentual do óleo essencial do Croton nepetaefoluis Baill. coletado em dois horários diferentes

\begin{tabular}{lcc}
\hline Constituintes & \multicolumn{2}{c}{ Horário de coleta } \\
\cline { 2 - 3 } & $6: 00 \mathrm{~h}$ & $13: 00 \mathrm{~h}$ \\
\hline$\alpha$-Pineno & 2,89 & 5,90 \\
Canfeno & 1,22 & 2,24 \\
$\beta$-Pineno & 1,22 & 2,78 \\
1,8 -Cineol & 0,83 & - \\
Cânfora & 3,53 & 5,94 \\
$\alpha$-Cubebeno & 1,27 & 1,43 \\
$\alpha$-Copaeno & 19,05 & 18,92 \\
$\beta$-Cubebeno & 4,37 & 3,07 \\
$\beta$-Elemeno & - & 5,41 \\
Metil-eugenol & 39,62 & 27,80 \\
E-Cariofileno & 5,42 & 4,75 \\
$\alpha$-Z-Bergamoteno & 1,70 & 1,00 \\
$\alpha$-Guaieno & 3,40 & 3,05 \\
$\alpha$-Humuleno & 1,12 & 0,88 \\
Biciclogermacreno & 5,03 & 7,90 \\
$\delta$-Cadineno & 4,44 & 4,08 \\
Óxido de cariofileno & 1,53 & 1,23 \\
\hline
\end{tabular}

Tabela 3. Composição química percentual do óleo essencial do Croton argyrophylloides Muell Arg. coletado em dois horários diferentes do dia

\begin{tabular}{lcc}
\hline Constituintes & \multicolumn{2}{c}{ Horário de coleta } \\
\cline { 2 - 3 } & $6: 00 \mathrm{~h}$ & $13: 00 \mathrm{~h}$ \\
\hline$\alpha$-Pineno & 14,23 & 4,48 \\
Sabineno & 1,37 & 1,66 \\
$\beta$-Pineno & 2,59 & 0,90 \\
para-Cimeno & 1,60 & 2,47 \\
$\beta$-Felandreno & - & 7,43 \\
1,8-Cineol & 8,15 & 4,58 \\
4-Terpineol & 1,99 & 2,34 \\
$\alpha$-Terpineol & 1,23 & 0,99 \\
$\beta$-Elemeno & 2,49 & 3,95 \\
$E$-Cariofileno & 2,35 & 10,13 \\
Aromadendreno & 1,84 & 2,30 \\
$\alpha$-Humuleno & - & 2,64 \\
Allo-aromadendreno & 1,47 & 2,11 \\
$\alpha$-Muuroleno & 2,28 & 2,24 \\
$\beta$-Selineno & 2,09 & 4,51 \\
$\alpha$-Selineno & - & 4,77 \\
$\beta$-E-Guaieno & 2,50 & 1,85 \\
$\gamma$-Cadineno & 3,86 & 3,01 \\
$\delta$-Cadineno & - & 2,95 \\
$E$-nerolidol & 2,27 & 1,63 \\
Espatulenol & 9,80 & 14,33 \\
Oxxido de cariofileno & 1,46 & 5,76 \\
Guaiol & - & 1,51 \\
\hline
\end{tabular}

significativamente na concentração de 100 em relação à de 1000 ppm, com exceção do óleo do C. nepetaefolius, que mostrou atividade semelhante nas três concentrações. Na concentração de 100 ppm, os óleos de C. zenhtneri e C. argyrophylloides dos dois horários foram mais ativos que os padrões. Na concentração de 500 ppm, todos os óleos foram semelhantes aos padrões considerados. Na concentração de 1000 ppm, C. zehntneri (6:00 h) e $C$. argyrophylloides $(13: 00 \mathrm{~h})$ foram semelhantes ao $\alpha$ tocoferol e mais ativos que o BHT; $C$. zehntneri $(13: 00 \mathrm{~h})$ e $C$. argyrophylloides $(6: 00 \mathrm{~h})$ foram mais ativos que o BHT; $C$. nepetaefolius dos dois horários foi menos ativo que os demais, no entanto foi semelhante ao BHT. A atividade antioxidante destes óleos deve-se provavelmente à presença entre seus constituintes químicos de vários componentes antioxidantes e alguns com atividade já relatada ${ }^{12}$, como $E$-anetol no $C$. zenhtneri e metileugenol no C. nepetaefolius. O óleo de C. argyrophylloides

Tabela 4. Resultado da atividade antioxidante dos óleos essenciais das espécies do gênero Croton pelo método TBARS

\begin{tabular}{lccc}
\hline Óleo (sigla) e padrões & \multicolumn{3}{c}{ Concentrações das Amostras / Valores de IA*(\%) } \\
\cline { 2 - 4 } & $1000 \mathrm{ppm}$ & $500 \mathrm{ppm}$ & $100 \mathrm{ppm}$ \\
\hline OECz $(6: 00 \mathrm{~h})$ & $52,33 \pm 0,27 \mathrm{Aa}$ & $27,18 \pm 3,08 \mathrm{Ba}$ & $22,83 \pm 5,38 \mathrm{Bab}$ \\
OECz $(13: 00 \mathrm{~h})$ & $41,30 \pm 2,75 \mathrm{Ab}$ & $23,68 \pm 6,00 \mathrm{Ba}$ & $21,26 \pm 3,58 \mathrm{Bab}$ \\
OECn $(6: 00 \mathrm{~h})$ & $25,92 \pm 2,33 \mathrm{Ac}$ & $17,57 \pm 3,99 \mathrm{Ba}$ & $11,92 \pm 4,10 \mathrm{Bcd}$ \\
OECn $(13: 00 \mathrm{~h})$ & $21,88 \pm 8,74 \mathrm{Ac}$ & $21,04 \pm 4,53 \mathrm{Aa}$ & $15,74 \pm 4,26 \mathrm{Abc}$ \\
OECa $(6: 00 \mathrm{~h})$ & $41,07 \pm 5,34 \mathrm{Ab}$ & $30,67 \pm 14,01 \mathrm{ABa}$ & $22,82 \pm 4,32 \mathrm{Bab}$ \\
OECa $(13: 00 \mathrm{~h})$ & $55,96 \pm 7,48 \mathrm{Aa}$ & $24,92 \pm 13,27 \mathrm{Ba}$ & $23,57 \pm 2,93 \mathrm{Ba}$ \\
BHT & $27,93 \pm 4,55 \mathrm{Ac}$ & $21,34 \pm 6,06 \mathrm{Aa}$ & $8,36 \pm 6,60 \mathrm{Bd}$ \\
$\alpha$ - tocoferol & $59,28 \pm 6,74 \mathrm{Aa}$ & $28,38 \pm 2,68 \mathrm{Ba}$ & $12,32 \pm 4,42 \mathrm{Ccd}$ \\
\hline
\end{tabular}

*IA = Índice de atividade antioxidante. Os valores entre parênteses referem-se aos desvios padrões das médias de quatro determinações. Letras maiúsculas diferentes representam diferenças significativas no índice de atividade (IA) entre as três concentrações do mesmo óleo. Letras minúsculas diferentes representam diferenças significativas no índice de atividade (IA) entre os diversos óleos em relação à mesma concentração. OECz: Óleo Essencial de Croton zehntneri; OECn: Óleo Essencial de Croton nepetaefolius; OECa: Óleo Essencial de Croton argyrophylloides. 
apresentou $\alpha$-pineno, $E$-cariofileno e 1,8-cineol, cujas atividades relatadas são baixas, mas provavelmente estão agindo sinergisticamente. $\mathrm{O}$ efeito sinergístico pode ser observado entre compostos fenólicos e não fenólicos, a exemplo do ácido ascórbico e lecitina ${ }^{21}$.

\section{CONCLUSÕES}

Os óleos essenciais do C. zenhtneri e C. nepetaefolius apresentam na sua constituição monoterpenóides e sesquiterpenóides, como também arilpropanóides; já o óleo do C. argyrophylloides apresenta monoterpenóides e sesquiterpenóides, mas não contém arilpropanóides. Os constituintes $E$-anetol, formiato de anisila, anisaldeído, metileugenol, 1,8-cineol, $\alpha$-pineno e espatulenol (Figura 1) foram os principais constituintes dos óleos essenciais estudados.

\section{Croton zenhtneri}

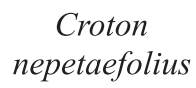

E-anetol<smiles>COc1ccc(C=O)cc1</smiles>
Anisaldeído $E$-anetol Anisaldido<smiles>C=CCc1ccc(OC)c(OC)c1</smiles>

Metil-eugenol<smiles>COc1ccc(COC=O)cc1</smiles>

Formiato de anisila

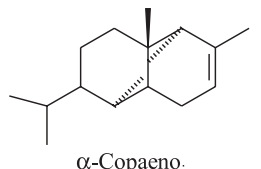

$\alpha$-Copaeno

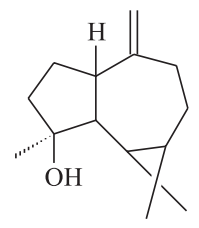

Espatulenol
Figura 1. Estruturas dos principais constituintes presentes nos óleos essenciais de espécies do gênero Croton estudadas

Entres estes compostos majoritários e alguns constituintes presentes em menor percentual, pode-se identificar, pelas informações da literatura ${ }^{12}$, vários de ação antioxidante relevante que provavelmente estão agindo sinergisticamente. O horário de coleta da planta deve ser levado em consideração na procura de um óleo com maior ação antioxidante, pois houve variação na constituição química dos óleos essenciais obtidos provocando, consequentemente, mudança nesta atividade. Em geral, os óleos do $C$. zenhtneri e $C$. argyrophylloides mostraram maior ação antioxidante que o $C$. nepetaefolius. A utilização popular das três espécies de Crotons fica valorizada pela verificação de sua ação antioxidante.

\section{AGRADECIMENTOS}

À Fundação Cearense de Apoio ao Desenvolvimento Científico e Tecnológico (FUNCAP) pelo apoio financeiro e bolsas de Iniciação Científica. Ao Conselho de Desenvolvimento Científico e Tecnológico $(\mathrm{CNPq})$ pelas bolsas de Iniciação Científica e ao Prof. A. A. Craveiro, do Parque de Desenvolvimento Tecnológico do Ceará (PADETEC) pela análise dos óleos essenciais.

\section{REFERÊNCIAS}

1. Desmachelier, C.; Romão, R. L.; Coussio, J.; Ciccia, G.; J. Ethnopharmacol. 1999, 67, 69.

2. Gottlieb, O.; Kaplan, M.; Borin, M.; Biodiversidade: Um Enfoque QuímicoBiológico, Ed. UFRJ: Rio de Janeiro, 1996.

3. Larson, R.; Phytochemistry 1988, 2, 969.

4. Spencer, C. M.; Cai, Y.; Martin, R.; Gaffney, S. H.; Goulding, P.; Magnolato, D.; Lilley, T. H.; Haslam; E.; Phytochemistry 1988, 27, 2397.

5. Yang, J.; Mau, J.; Ko, P.; Huang, L.; Food Chem. 2000, 71, 249.

6. Weng, X. C.; Wang, W.; Food Chem. 2000, 71, 489.

7. Jayaprakasha, G. K.; Singh, R. P.; Sakariah, K. K.; Food Chem. 2001, 73, 285.

8. Loliger, J. Em Free radicals in food additivies; Auroma, I. O.; Halliwell, B., eds.; Taylor \& Francis: London, 1991.

9. Bracco, U.; Loliger, J.; Viret, J. I.; J. Am. Oil Chem. Soc. 1981, 58, 686.

10. Tisserand, R.; Balacs, T.; Essential Oil Safety: A Guide for Health Care Professionals, Churchill Livingstone: London, 1999.

11. Mantle, D.; Anderton, J. G.; Falkous, G.; Barnes, M.; Jones, P.; Perry, E. K.; Comp. Biochem. Physiol., Part B: Biochem. Mol. Biol. 1998, 121, 385.

12. Ruberto, G.; Baratta, M. T.; Food Chem. 2000, 69, 167.

13. Catunda Jr., F. E. A.; Luciano, J. H. S.; Morais, S. M.; Rev. Ciência e Tecnol. da UECE 2002, 4, 23.

14. Craveiro, A. A.; Andrade, C. H. S.; Matos, F. J. A.; Alencar, J. W.; J. Agric. Food Chem. 1978, 26, 773.

15. Oliveira, A. C.; Leal-Cardoso, J. H.; Santos, C. F.; Morais, S. M.; Coelhode-Souza, A. N.; Braz. J. Med. Biol. Res. 2001, 34, 1471.

16. Abdom, A. P. V.; Leal-Cardoso, J. H.; Coelho-de-Souza, A. N.; Morais, S. M.; Santos, C.F.; Braz. J. Med. Biol. Res. 2002, 35, 1215.

17. Craveiro, A. A.; Matos, F. J. A.; Alencar, J. W.; J. Chem. Educ. 1976, 53, 652.

18. Adams, R. P.; Identification of Essential Oil Components by Gas Chromatography / Quadrupole Mass Spectroscopy; Allured Publishing Corporation: USA, 2001.

19. Kishida, E.; Kamura, A.; Tokumaru, S.; Oribe, M.; Igushi, H.; Kojo, S.; J. Agric. Food Chem. 1993, 41, 2707.

20. SAS user's guide: statistics, SAS Institute Inc.: Cary, NC, 2001.

21. Melo, E. A.; Guerra, N. B.; Bol. SBCTA 2002, 36, 1. 